\title{
Subchondroplasty in the Treatment of Bone Marrow Lesions of the Knee: Preliminary Experience on First 15 Patients
}

\author{
Stefano Pasqualotto ${ }^{1}$ Andrea Vincenzo Sgroi ${ }^{1}$ Araldo Causero ${ }^{2}$ Paolo Di Benedetto ${ }^{2}$ (1) \\ Claudio Zorzi ${ }^{1}$
}

${ }^{1}$ Divisione di Ortopedia e Traumatologia, IRCCS Ospedale Classificato Equiparato Sacro Cuore - Don Calabria, Negrar (Verona), Italy

${ }^{2}$ DAME Università degli studi di Udine, Clinica Ortopedica, Azienda

Address for correspondence Stefano Pasqualotto, MD, Divisione di Ortopedia e Traumatologia, IRCCS Ospedale Classificato Equiparato Sacro Cuore - Don Calabria, Via don A. Sempreboni, 5 - 37024 Negrar Sanitaria Universitaria Friuli Centrale, Udine, Italy (Verona), Italy (e-mail: stefano.pasqualotto20@gmail.com).

Joints 2019;7:174-181.

\section{Abstract \\ Keywords \\ - bone marrow lesions \\ - subchondroplasty \\ - knee osteoarthritis \\ - calcium phosphate bone substitute}

Purpose The aim of this prospective study was to assess the effectiveness in terms of pain relief and functional improvement of the Subchondroplasty procedure in the treatment of osteoarthritis-related bone marrow lesions (BMLs) of the knee.

Methods The study included first 15 consecutive patients undergone to Subchondroplasty procedure for the treatment of chronic degenerative BMLs in which previous conservative treatment have failed. Western Ontario and McMaster Universities Osteoarthritis Index (WOMAC) scores, Knee Injury and Osteoarthritis Outcome Scores (KOOS), and visual analog scale (VAS) pain scores were obtained preoperatively and at 1,6 , and 12 months of follow-up.

Results WOMAC scores significantly improved from $39.7 \pm 20.2$ before surgery to $26.8 \pm 16.1$ at the 1 -month follow-up $(p=0.045)$. A further significant improvement to $15.5 \pm 12.7(p=0.02)$ and to $8.6 \pm 3.1(p<0.01)$ was obtained both at 6 -month and at 1-year follow-up. KOOS scores improved significantly from $47.5 \pm 16.6$ before surgery to $65.4 \pm 14.9$ at 1 month $(p=0.013)$ and to $80.4 \pm 15.1$ at 6 -month follow-up $(p=0.01)$. A further improvement to $85.6 \pm 15.1$ was recorded 1 year postoperatively, although nonsignificant. VAS score showed a significant improvement from $55.8 \pm 20.5$ preoperatively to $36.2 \pm 16.9$ at 1 month $(p=0.008)$ and to $18.2 \pm 17.3$ at 6-month follow-up $(p=0.005)$. This further improved to $12.8 \pm 17.9$ at 1-year followup, although not significantly.

Conclusion Subchondroplasty procedure represents a safe and valid surgical option in the treatment of osteoarthritis-related BMLs of the knee, providing an improvement in terms of pain relief and functional recovery. Longer studies are required to evaluate how long these improvements may last.

Level of Evidence Therapeutic case-series, Level IV study. received

February 7, 2020 accepted after revision

April 19, 2021

published online

June 18, 2021
DOI https://doi.org/ $10.1055 / \mathrm{s}-0041-1730984$ ISSN 2282-4324.

\footnotetext{
(c) 2021. The Author(s).

This is an open access article published by Thieme under the terms of the Creative Commons Attribution-NonDerivative-NonCommercial-License, permitting copying and reproduction so long as the original work is given appropriate credit. Contents may not be used for commercial purposes, or adapted, remixed, transformed or built upon. (https://creativecommons.org/ licenses/by-nc-nd/4.0/) Georg Thieme Verlag KG, Rüdigerstraße 14, 70469 Stuttgart, Germany
} 


\section{Introduction}

Knee osteoarthritis (OA) is a degenerative process, which affects not only the articular cartilage but the entire joint, including synovium, ligaments, menisci, and periarticular muscles and tendons. ${ }^{1,2}$ This process influences also the subchondral bone (SCB) and alterations are seen both from mechanical, morphological, and histochemical point of view. $^{3}$

SCB plays therefore a key role in the pathophysiology and progression of $\mathrm{OA}$. The presence of bone marrow lesions (BMLs) in the setting of knee OA was correlated with pain, clinical deterioration, decreased quality of life, ${ }^{4-7}$ and with 6.5- and 7-fold greater probabilities of OA progression. ${ }^{8}$ Moreover, the presence of BMLs was associated with an accelerated progression to total knee arthroplasty (TKA). ${ }^{9-11}$

BMLs, defined as high-signal-intensity alterations detected on magnetic resonance (MR) fluid-sensitive sequences [T2/proton density with fat suppression and short tau inversion recovery] with a decreased bone marrow signal intensity on T1-weighted image, are a common feature of knee OA. ${ }^{12}$ Despite originally defined as bone marrow edema, several histological studies revealed that edema in the region of BML is minimal. ${ }^{13,14}$ In the osteoarthritic process, these areas are rather characterized by trabecular bone microdamages and microcracks ${ }^{15-17}$ associated with altered bone mineralization and increased bone remodeling. ${ }^{18}$ Moreover, vascular structure abnormalities, ${ }^{14,15}$ zone of fatty marrow, necrosis, and bone marrow fibrosis were detected. $^{13,19,20}$

In recent years, therefore, interests were focused on interventions directed toward the $\mathrm{SCB}$ to prevent or reverse BMLs. Among these, Subchondroplasty (SCP) (Zimmer Knee Creations, West Chester, Pennsylvania, United States) is a procedure that utilizes an injectable, synthetic, calcium phosphate (CaP) bone void filler to treat chronic BMLs in which previous conservative treatment failed. ${ }^{21}$ The goal of this procedure is to improve the structural properties of the affected SCB and stimulate SCB remodeling, with the aim of preventing bone collapse and OA progression. ${ }^{21,22}$

Purpose of this preliminary study was to evaluate clinical and functional outcomes of the treatment of OA-related knee BML with SCP combined with arthroscopy.

\section{Methods}

This prospective 1-year follow-up case-series was conducted on first 15 consecutive patients prospectively enrolled from February 2018 to October 2018 to undergo SCP for the treatment of OA-related BML of the knee. The study was approved by the institutional review board and written informed consent was obtained from each patient included.

Inclusion criteria were age between 40 and 75 years, atraumatic and localized knee pain for at least 6 months with failure of previous conservative treatments, and an MR imaging (MRI) showing a BML located in the subchondral

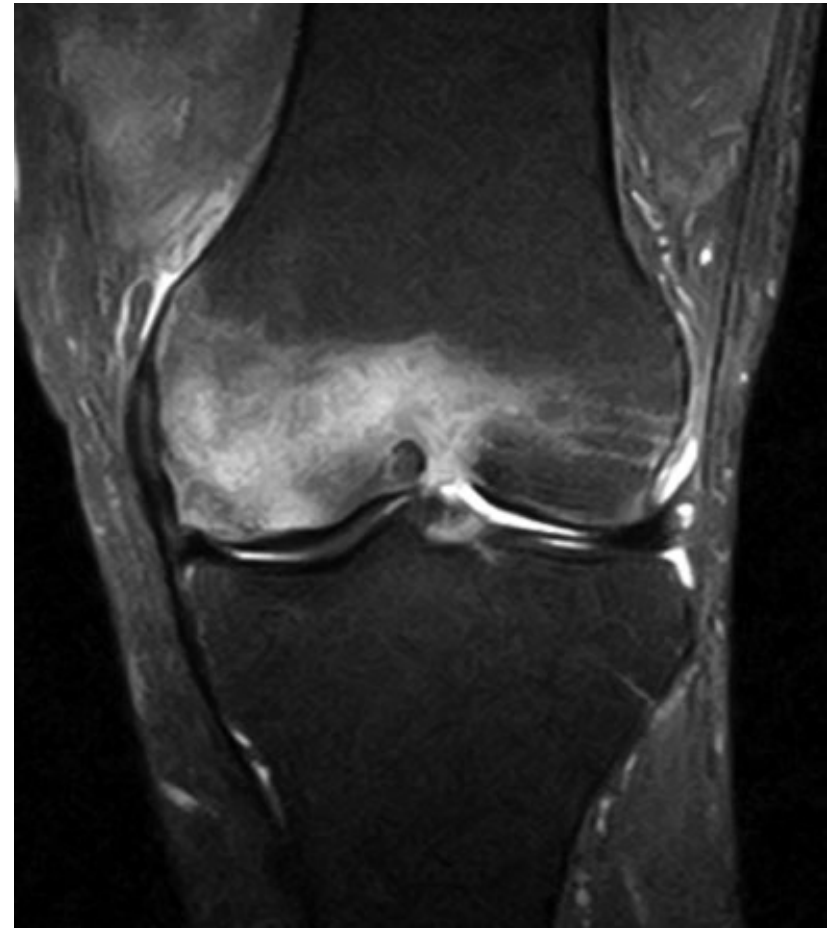

Fig. 1 T2-weighted magnetic resonance imaging of patient's left knee before surgery showing bone marrow lesion of the medial femoral condyle.

region of the tibial plateau and/or femoral condyle, in correspondence to the location of knee pain (-Fig. 1 ).

Exclusion criteria were: generalized knee pain, known autoimmune disorders, active malignancies or infections, OA $>$ grade 3 according to Kellgren-Lawrence, ${ }^{23}$ patellofemoral $\mathrm{OA}>$ grade 3 according to Iwano classification ${ }^{24}$ associated with symptoms related to patellofemoral joint, and varus or valgus malalignment of the lower limb more than 10 degrees.

MRI images were analyzed with RadiAnt DICOM Viewer (Medixant, Poznan, Poland) and BMLs were mapped in axial, sagittal, and coronal view to measure the distance from the joint line and the nearest cortex and to plan the direction of the cannula.

Anteroposterior, lateral, and Merchant views were performed to grade osteoarthritic changes whereas full-length lower limb X-rays were performed to assess lower limb alignment.

All the patients underwent the same clinical and radiological evaluations and were assessed preoperatively and at 1, 6, and 12 months with Western Ontario and McMaster Universities Osteoarthritis Index (WOMAC) and Knee injury and Osteoarthritis Outcome Score (KOOS) whereas pain was measured using the visual analog scale (VAS).

All the patients underwent X-rays evaluations immediately after the procedure and at 1, 6, and 12 months postoperatively whereas MRI evaluations were performed at 6 and 12 months. Intra- and postoperative complications were also recorded. 


\section{Surgical Technique and Postoperative Management}

After the preoperative planning, the SCP procedure was performed with the patient in supine position under spinal anesthesia.

According to preoperative planning, the entry point of the cannula was marked on the skin with the aid of fluoroscopy on both frontal and lateral view. A skin incision was then performed and the cannula introduced until the bone. A second check with the aid of the fluoroscopy was done to identify the proper entry point. The cannula was then introduced and drilling was continued until it reached the lesion. A fluoroscopic check was done to control the exact position of the cannula and to assess that all the three holes of the side-delivery cannula were deep to the cortex and oriented in the desired direction.

The AccuFill Bone Substitute Material (ETEX Corporation, Cambridge, Massachusetts, United States) was then prepared until the proper viscosity was reached and introduced into the injured area, checking its distribution with the aid of fluoroscopy (-Fig. 2).

After the SCP procedure, arthroscopy was performed to check the intra-articular leakage of the bone substitute and to evaluate and address intra-articular pathology such as chondral flaps, loose bodies, and degenerative meniscal tears.

The postoperative management consisted of partial weight-bearing with the aid of crutches for 1 week and then full weight-bearing was allowed without any restrictions in terms of range of motion. Return to daily-life activities was encouraged as soon as tolerated.

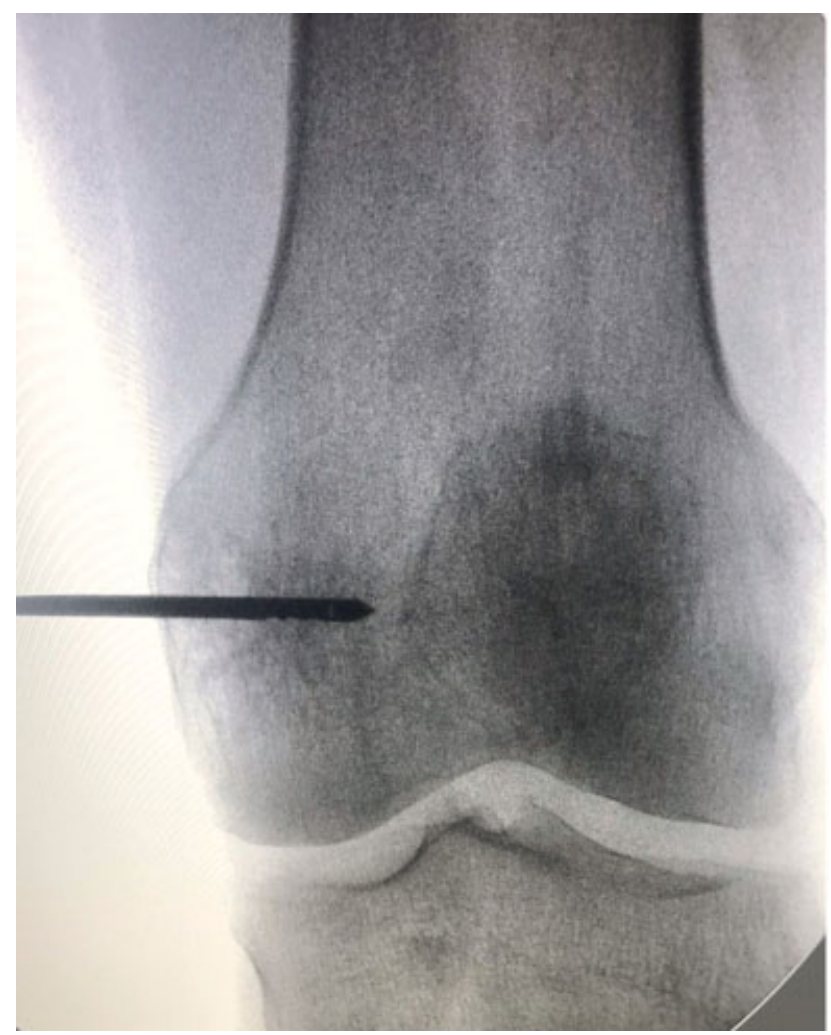

Fig. 2 Intraoperative fluoroscopic anteroposterior (AP) image showing the placement of the cannula and the diffusion of the calcium phosphate $(\mathrm{CaP})$ in the medial femoral condyle.

\section{Statistical Analysis}

Statistical analyses were performed using RStudio v. 1.1.383 software (RStudio, Inc., Boston, Massachusetts, United States) and a $p$-value of $\leq 0.05$ was considered statistically significant. The Kolmogorov-Smirnov and Shapiro-Wilk tests were performed to assess the normality of the distributions. Descriptive statistics were calculated: paired Student's $t$-test and the Wilcoxon matched-pairs signed rank test were used to test for significant differences between baseline and outcome score measurements.

\section{Results}

Ten of the 15 patients were male whereas 5 were female with a mean age at the time of surgery of $53.6 \pm 9.4$ years (range: 40-72). In seven cases, BML was located at the medial tibial plateau, in three cases at the medial femoral condyle, and in five cases both medial tibial plateau and medial femoral condyle were involved. At the preoperative X-rays evaluations, two patients had a grade 1 tibiofemoral OA, eight patients had a grade 2, and five patients had a grade 3 according to Kellgren-Lawrence, ${ }^{23}$ whereas according to Iwano classification ${ }^{24}$ five patients did not show any sign of patellofemoral OA, seven patients had grade 1 , and three patients had grade 2 . Six of the 15 patients have had previous surgery on the same knee; among these, four have undergone medial meniscectomy, one patient to an anterior cruciate ligament $(\mathrm{ACL})$ reconstruction associated with a medial meniscectomy, and one patient has had a tibial tubercle osteotomy (-Table $\mathbf{1}$ ).

Table 1 Demographic data of patients treated with subchondroplasty

\begin{tabular}{|c|c|}
\hline No of patients & 15 \\
\hline Mean age & 53.6 years (range $40-72$ ) \\
\hline Sex & $\begin{array}{l}10 \text { male } \\
5 \text { female }\end{array}$ \\
\hline Side of knee (\%) & $\begin{array}{l}9 \text { right (60) } \\
6 \text { left (40) }\end{array}$ \\
\hline Location of BML (\%) & $\begin{array}{l}7 \text { MTP (46.6) } \\
3 \text { MFC (20) } \\
5 \text { MTP and MFC (33.3) }\end{array}$ \\
\hline Kellgren-Lawrence & $\begin{array}{l}\text { Grade I: } 2 \text { patients }(13.33 \%) \\
\text { Grade II: } 8 \text { patients }(53.33 \%) \\
\text { Grade III: } 5 \text { patients }(33.33 \%)\end{array}$ \\
\hline Iwano & $\begin{array}{l}\text { Grade } 0: 5 \text { patients }(33.33 \%) \\
\text { Grade I: } 7 \text { patients }(46.66 \%) \\
\text { Grade II: } 3 \text { patients }(20 \%)\end{array}$ \\
\hline $\begin{array}{l}\text { Previous surgery (\%) } \\
\text { Partial medial } \\
\text { meniscectomy } \\
\text { ACL reconstruction } \\
\text { Tibial tubercle } \\
\text { osteotomy }\end{array}$ & $\begin{array}{l}6 \text { patients }(40) \\
4 \text { patients } \\
1 \text { patient } \\
1 \text { patient }\end{array}$ \\
\hline
\end{tabular}

Abbreviations: $\mathrm{ACL}$, anterior cruciate ligament; $\mathrm{BML}$, bone marrow lesion; MFC, medial femoral condyle; MTP, medial tibial plateau. 
Table 2 Clinical scores preoperative and at 1 month, 6 months, and 1 year postoperative

\begin{tabular}{|l|l|l|l|l|}
\hline Clinical score & $\begin{array}{l}\text { Preop } \\
\text { Mean } \pm \text { SD }\end{array}$ & $\begin{array}{l}\mathbf{1} \text { mo } \\
\text { Mean } \pm \text { SD }\end{array}$ & $\begin{array}{l}\mathbf{6} \text { mo } \\
\text { Mean } \pm \text { SD }\end{array}$ & $\begin{array}{l}\mathbf{y} \\
\text { Mean } \pm \text { SD }\end{array}$ \\
\hline WOMAC & $39.7 \pm 20.2$ & $26.8 \pm 16.1$ & $15.5 \pm 12.7$ & $8.6 \pm 3.1$ \\
\hline VAS & $55.8 \pm 20.5$ & $36.2 \pm 16.9$ & $18.2 \pm 17.3$ & $12.8 \pm 17.9$ \\
\hline KOOS-Pain & $56.6 \pm 19.6$ & $61.3 \pm 13.8$ & $81.2 \pm 14.6$ & $88.1 \pm 17.6$ \\
\hline KOOS-Symptoms & $56.5 \pm 18.9$ & $68.7 \pm 14.6$ & $81.2 \pm 14.1$ & $90.9 \pm 11.3$ \\
\hline KOOS-ADL & $64.1 \pm 23.3$ & $70.4 \pm 16.3$ & $85.6 \pm 11.5$ & $92.0 \pm 13.6$ \\
\hline KOOS-Sports & $23.7 \pm 23.2$ & $44.8 \pm 24.8$ & $66.5 \pm 23.9$ & $66.9 \pm 20.9$ \\
\hline KOOS-QoL & $30.3 \pm 17.1$ & $38.6 \pm 21.5$ & $65.3 \pm 22.8$ & $70.0 \pm 24.0$ \\
\hline
\end{tabular}

Abbreviations: ADL, activity of daily life; KOOS, Knee Injury and Osteoarthritis Outcome Scores; QoL, quality of life; SD, standard deviation; VAS, visual analog scale; WOMAC, Western Ontario and McMaster Universities Osteoarthritis Index.

Preoperative evaluations showed a mean WOMAC of $39.7 \pm 20.2$, a mean KOOS total score of $47.5 \pm 16.6$, and a mean VAS score of $55.8 \pm 20.5 \mathrm{~mm}$.

At follow-up assessments, significant increases were recorded in both WOMAC score, KOOS subscales, and VAS scale as detailed below (-Table $\mathbf{2}$ ).

In comparison to preoperative values, WOMAC score 1 month postoperatively was $26.88 \pm 16.1$, showing a significant improvement $(p=0.045)$ which was maintained at 6-month follow-up when a mean value of $15.5 \pm 12.7$ was obtained $(p=0.02)$ and also at 1 -year follow-up when a mean value of $8.6 \pm 3.1$ was recorded $(p=0.004)$.

KOOS score recorded 1 month postoperatively revealed a significant improvement in comparison to preoperative values $(p=0.013)$ and this improvement was also recorded 6 months after the procedure when a mean value of $80.4 \pm 15.1$ ( $p=0.01)$ was obtained. KOOS at 1-year followup revealed a little improvement with a mean score of $85.6 \pm 15.1$ but no statistically significant differences were found with the 6-month mean value. -Fig. 3 shows the distribution of KOOS subscales at the different evaluations.
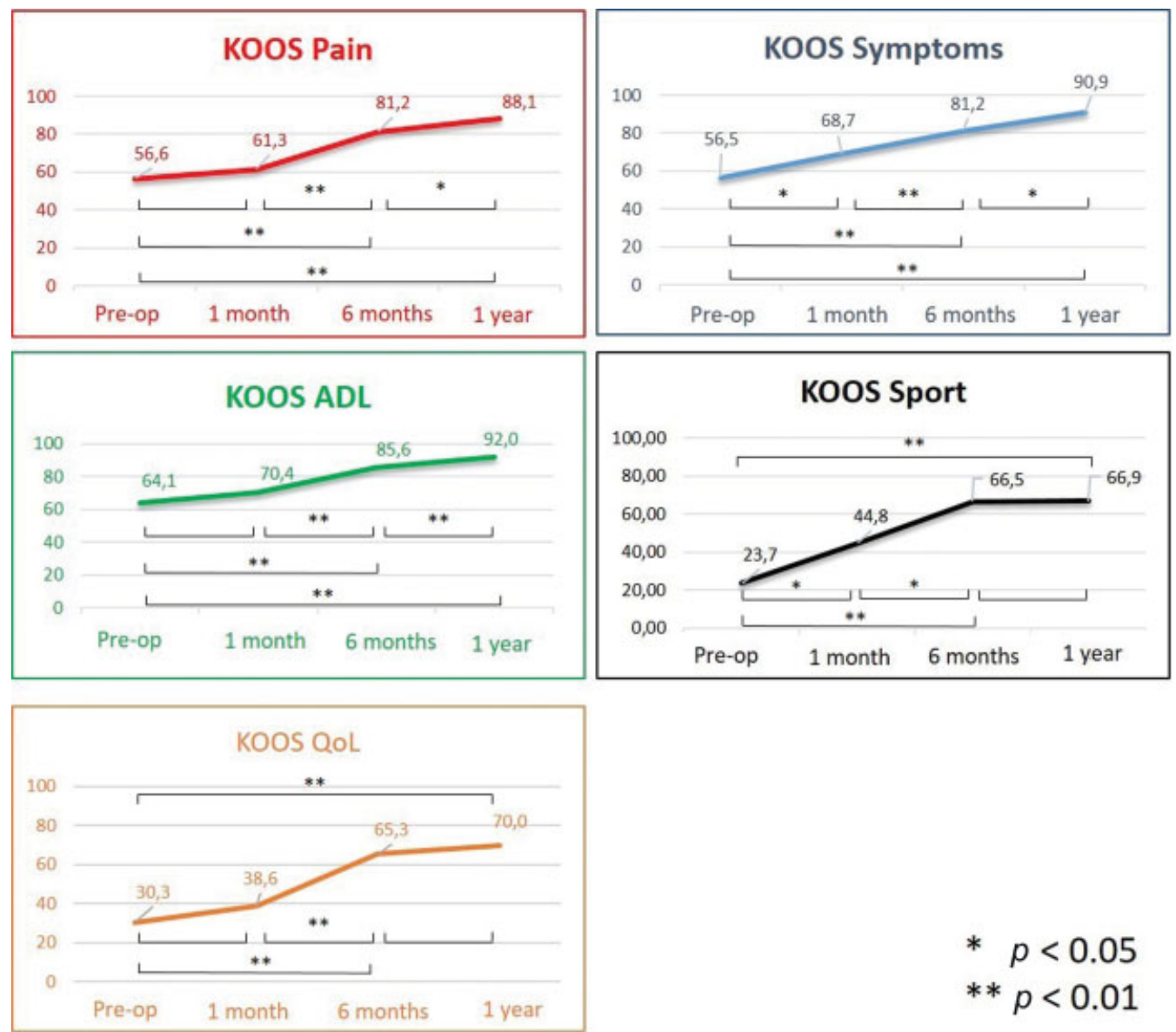

Fig. 3 Line graphs of Knee Injury and Arthritis Outcome Score subscales preoperatively and at 1-, 6-, and 12-month follow-up. 


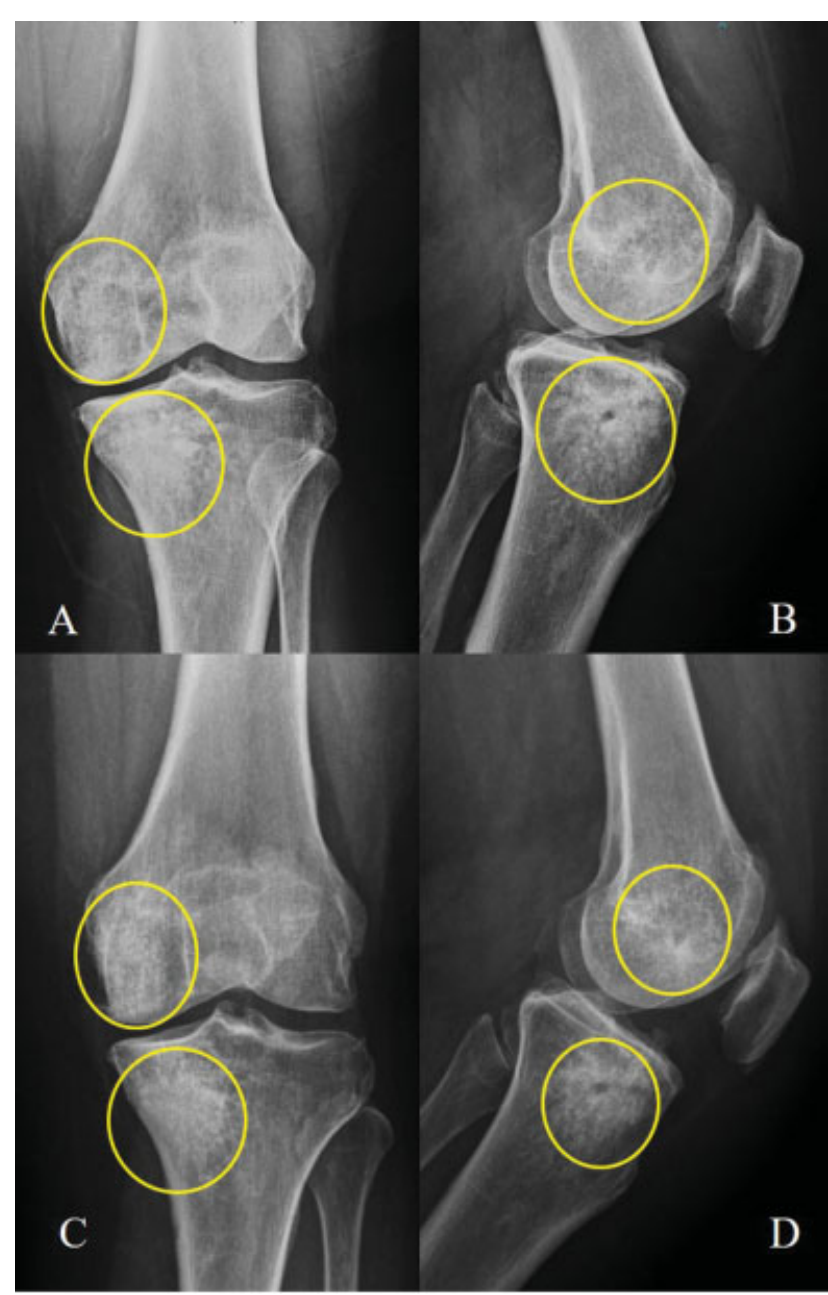

Fig. 4 (A-D) Anteroposterior and lateral postoperative X-rays showing the increased density of the injection site (yellow circles) 1 month (A, B) and 6 months (C, D) after Subchondroplasty (SCP).
In comparison to preoperative value, mean VAS significantly decreased to $36.2 \pm 16.9 \mathrm{~mm}$ after 1 month $(p=0.008)$ and a further improvement was seen at 6-month follow-up with a mean value of $18.2 \pm 17.3 \mathrm{~mm}(p=0.005)$. At 1 -year follow-up, VAS score showed a little progression with a mean value of $12.8 \pm 17.9$ without reaching a statistically significant difference from the value obtained at 6 months.

$\mathrm{X}$-rays imaging in the postoperative period revealed increased density in the site where the $\mathrm{CaP}$ were introduced (-Fig. 4A-D) whereas MR images showed an area of low signal intensity at the injection site (-Fig. 5B, C).

In terms of complications, only one patient underwent an intraoperative complication with leakage of part of the CaP outside the tibia, which was immediately removed by enlarging the skin incision without any adverse outcome for the patient. None of the 15 patients showed a progression of the disease with a collapse of the joint surfaces.

\section{Discussion}

The most important finding of this prospective study was that SCP was able to provide a significant improvement in terms of pain relief and knee functional capacity in patients with chronic OA-related BMLs of the knee in which previous conservative treatment have failed.

At 1-year follow-up, pain improvement was 43 points on the VAS scale, in which the minimal clinically important differences (MCIDs) is $20 \mathrm{~mm} .{ }^{25}$ Similarly, mean functional improvement on the WOMAC was 31.1 points for which MCID is $9.1,{ }^{25}$ whereas KOOS showed a mean improvement of 38.1 points with a MCID of 8 to 10 points. $^{26}$

Traditionally, treatment of BMLs was based on nonsteroidal anti-inflammatory drugs, analgesics, and restricted weight-bearing especially in case of small lesions (< $\left.3.5 \mathrm{~cm}^{2}\right){ }^{27,28}$ More recently, pulsed electromagnetic fields ${ }^{29,30}$ and extracorporeal shockwave therapy ${ }^{31-33}$

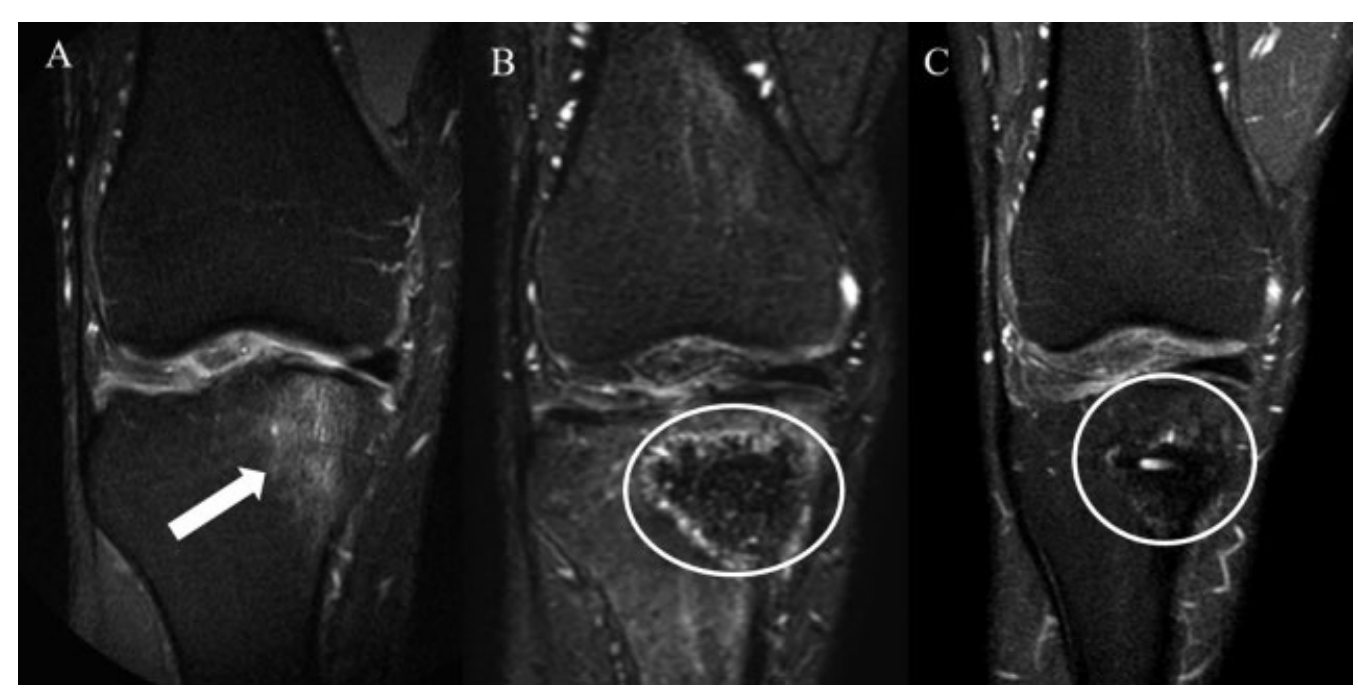

Fig. 5 (A-C) Fluid-sensitive magnetic resonance images showing the evolution from (A) preoperative bone marrow lesion (BML) at the medial tibial plateau (white arrow) to (B) 6-month image which show an hypointense area corresponding to the injected calcium phosphate (CaP) with a surrounding hyperintense rim (white circle). In 1-year image (C) CaP is assuming the intensity of the surrounding bone even though persist a mild hypointense area (white circle). 
achieved good results in the treatment of BMLs of the knee, though evidences are limited and long-term results have not been investigated yet.

Likewise, intravenous bisphosphonates ${ }^{34-36}$ and prostacyclin analogs ${ }^{37-39}$ showed promising results in the treatment of OA-related BMLs and early stage osteonecrosis in terms of both functional recovery and BML area reduction. However, further studies are needed to clarify indications, safety, and posology of these drugs.

In case of failure of conservative treatment, surgical approach is recommended and SCP is a recently developed procedure in which a bone substitute material composed of $\mathrm{CaP}$ is employed to treat chronic nonhealing BMLs. ${ }^{40}$ The results of the present study are largely consistent with some of the other studies that evaluated the effect of SCP in the treatment of BMLs of the knee.

Sharkey et $\mathrm{al}^{21}$ were the first to describe the use of SCP in a 51-year-old woman with a chronic OA-related BML associated with disabling knee pain resistant to previous conservative treatment. The follow-up at 31 months showed excellent clinical and functional outcomes and a complete resolution of subchondral BML.

Farr and Cohen ${ }^{41}$ in a cohort of 59 patients with chronic localized knee pain associated with subchondral BML reported a significant pain reduction and functional improvement in $75 \%$ of patients at a mean follow-up of 14.8 months after SCP. However, the study showed a failure rate of $25 \%$ with 15 out of 59 patients who underwent a unicompartmental knee arthroplasty or a TKA at a mean follow-up of 10.1 months.

Chatterjee et $\mathrm{al}^{42}$ in a retrospective study on 22 patients with OA-related BMI reported a significant functional improvement $(p<0.001)$ on the KOOS and Tegner-Lysholm Knee Scoring Scale at a minimum follow-up of 6 months after SCP associated with knee arthroscopy. Despite these promising results, the authors advised against the use of SCP since 10 out of 22 patients were considered clinical failure according to clinical outcome categories of Tegner-Lysholm Knee Scoring Scale, which was originally developed to evaluate ACL reconstruction and, therefore, not indicated in the evaluation of the success rate of this treatment. ${ }^{43}$

Cohen and Sharkey, ${ }^{22}$ in a study on 66 patients, demonstrated at 2-year follow-up a significant improvement of 4.5 point on the VAS scale and of 17.8 points on the International Knee Documentation Committee Subjective Knee Evaluation Form. Moreover, at 2-year follow-up, the authors registered a failure rate of $30 \%$, since 18 patients underwent TKA. Cohen's failure rate was higher in comparison with that of the current study. However, it should be noted that patient selection was different with Cohen's cohort including patients with moderate to severe $\mathrm{OA}$, initially presented for arthroplasty consultation who, to the contrary, were excluded from the present study.

Bonadio et $\mathrm{al}^{44}$ in a case-series of 5 patients with OArelated BMLs treated with SCP, reported a significant pain relief with a reduction of 7.2 points on the VAS scale and a significant functional improvement of 32.8 points on the
KOOS. However, these results referred to a short-term follow-up of 24 weeks.

More recently, Chua et $\mathrm{al}^{45}$ reported very good results in a cohort of 12 patients with chronic localized knee pain associated with a traumatic or degenerative subchondral insufficiency fracture detected with MRI. The authors demonstrated immediate postoperative pain relief that persisted up to 1 year with a reduction of 5.4 points on the VAS scale. Moreover, as in the present study, a significant functional recovery with an early return to activity of daily living was evidenced by an improvement of 34.7 points on the KOOS and of 33.5 on the WOMAC scale at 1-year follow-up.

Despite the good results, the present study has some limitations. First, the follow-up period is limited to 1 year whereas a study with a longer follow-up would be able to better evaluate the durability of the improvement. Second, arthroscopic treatment of intra-articular pathology could represent an important limitation since the relative contribution of each procedure could not be evaluated. However, it should be noted that several studies in patients with knee OA showed that arthroscopic debridement was not able to provide a durable relief of OA symptoms. ${ }^{46-48}$ Third, we acknowledge that our results refers to a small, nonrandomized cohort without a control group.

In conclusion, based on the results of the present study and literature considerations, it is possible to claim that SCP in the treatment of OA-related BMLs provide a significant improvement in terms of pain relief and functional recovery although on short-term follow-up. This safe, mini-invasive, and joint-sparing procedure could represent a valid secondline therapeutic option in the treatment of chronic OArelated BMLs in which previous conservative treatment has failed. Anyway, randomized controlled studies with broader cohort and longer follow-up are needed to confirm these promising results and to detect real benefits that SCP could provide in the treatment of BMLs of the knee.

Conflict of Interest

None declared.

\section{References}

1 Felson DT. An update on the pathogenesis and epidemiology of osteoarthritis. Radiol Clin North Am 2004;42(01):1-9

2 Loeser RF, Goldring SR, Scanzello CR, Goldring MB. Osteoarthritis: a disease of the joint as an organ. Arthritis Rheum 2012;64(06): 1697-1707

3 Singh V, Oliashirazi A, Tan T, Fayyad A, Shahi A. Clinical and pathophysiologic significance of MRI identified bone marrow lesions associated with knee osteoarthritis. Arch Bone Jt Surg 2019;7(03):211-219

4 Felson DT, Chaisson CE, Hill CL, et al. The association of bone marrow lesions with pain in knee osteoarthritis. Ann Intern Med 2001;134(07):541-549

5 Link TM, Steinbach LS, Ghosh S, et al. Osteoarthritis: MR imaging findings in different stages of disease and correlation with clinical findings. Radiology 2003;226(02):373-381

6 Lo GH, McAlindon TE, Niu J, et al; OAI Investigators Group. Bone marrow lesions and joint effusion are strongly and independently associated with weight-bearing pain in knee osteoarthritis: data 
from the osteoarthritis initiative. Osteoarthritis Cartilage 2009; 17(12):1562-1569

7 Roemer FW, Nevitt MC, Felson DT, et al. Predictive validity of within-grade scoring of longitudinal changes of MRI-based cartilage morphology and bone marrow lesion assessment in the tibiofemoral joint-the MOST study. Osteoarthritis Cartilage 2012;20 (11):1391-1398

8 Felson DT, McLaughlin S, Goggins J, et al. Bone marrow edema and its relation to progression of knee osteoarthritis. Ann Intern Med 2003;139(5 Pt 1):330-336

9 Tanamas SK, Wluka AE, Pelletier JP, et al. Bone marrow lesions in people with knee osteoarthritis predict progression of disease and joint replacement: a longitudinal study. Rheumatology (Oxford) 2010;49(12):2413-2419

10 Raynauld JP, Martel-Pelletier J, Haraoui B, et al; Canadian Licofelone Study Group. Risk factors predictive of joint replacement in a 2-year multicentre clinical trial in knee osteoarthritis using MRI: results from over 6 years of observation. Ann Rheum Dis 2011;70 (08):1382-1388

11 Scher C, Craig J, Nelson F. Bone marrow edema in the knee in osteoarthrosis and association with total knee arthroplasty within a three-year follow-up. Skeletal Radiol 2008;37(07):609-617

12 Kon E, Ronga M, Filardo G, et al. Bone marrow lesions and subchondral bone pathology of the knee. Knee Surg Sports Traumatol Arthrosc 2016;24(06):1797-1814

13 Zanetti M, Bruder E, Romero J, Hodler J. Bone marrow edema pattern in osteoarthritic knees: correlation between MR imaging and histologic findings. Radiology 2000;215(03):835-840

14 Saadat E, Jobke B, Chu B, et al. Diagnostic performance of in vivo 3T MRI for articular cartilage abnormalities in human osteoarthritic knees using histology as standard of reference. Eur Radiol 2008; 18(10):2292-2302

15 Muratovic D, Findlay DM, Cicuttini FM, Wluka AE, Lee YR, Kuliwaba JS. Bone matrix microdamage and vascular changes characterize bone marrow lesions in the subchondral bone of knee osteoarthritis. Bone 2018;108:193-201

16 Alliston T, Hernandez CJ, Findlay DM, Felson DT, Kennedy OD. Bone marrow lesions in osteoarthritis: what lies beneath. J Orthop Res 2018;36(07):1818-1825

17 Taljanovic MS, Graham AR, Benjamin JB, et al. Bone marrow edema pattern in advanced hip osteoarthritis: quantitative assessment with magnetic resonance imaging and correlation with clinical examination, radiographic findings, and histopathology. Skeletal Radiol 2008;37(05):423-431

18 Hunter DJ, Lavalley M, Li J, et al. Biochemical markers of bone turnover and their association with bone marrow lesions. Arthritis Res Ther 2008;10(04):R102

19 Leydet-Quilici H, Le Corroller T, Bouvier C, et al. Advanced hip osteoarthritis: magnetic resonance imaging aspects and histopathology correlations. Osteoarthritis Cartilage 2010;18(11): 1429-1435

20 Plenk H Jr, Hofmann S, Eschberger J, et al. Histomorphology and bone morphometry of the bone marrow edema syndrome of the hip. Clin Orthop Relat Res 1997;(334):73-84

21 Sharkey PF, Cohen SB, Leinberry CF, Parvizi J. Subchondral bone marrow lesions associated with knee osteoarthritis. Am J Orthop 2012;41(09):413-417

22 Cohen SB, Sharkey PF. Subchondroplasty for treating bone marrow lesions. J Knee Surg 2016;29(07):555-563

23 Kellgren JH, Lawrence JS. Radiological assessment of osteo-arthrosis. Ann Rheum Dis 1957;16(04):494-502

24 Iwano T, Kurosawa H, Tokuyama H, Hoshikawa Y. Roentgenographic and clinical findings of patellofemoral osteoarthrosis. With special reference to its relationship to femorotibial osteoarthrosis and etiologic factors. Clin Orthop Relat Res 1990;(252): 190-197

25 Tubach F, Ravaud P, Baron G, et al. Evaluation of clinically relevant changes in patient reported outcomes in knee and hip osteoar- thritis: the minimal clinically important improvement. Ann Rheum Dis 2005;64(01):29-33

26 Roos EM, Lohmander LS. The Knee injury and Osteoarthritis Outcome Score (KOOS): from joint injury to osteoarthritis. Health Qual Life Outcomes 2003;1:64

27 Lotke PA, Abend JA, Ecker ML. The treatment of osteonecrosis of the medial femoral condyle. Clin Orthop Relat Res 1982;(171): 109-116

28 Yates PJ, Calder JD, Stranks GJ, Conn KS, Peppercorn D, Thomas NP. Early MRI diagnosis and non-surgical management of spontaneous osteonecrosis of the knee. Knee 2007;14(02):112-116

29 Gobbi A, Lad D, Petrera M, Karnatzikos G. Symptomatic early osteoarthritis of the knee treated with pulsed electromagnetic fields: two-year follow-up. Cartilage 2014;5(02):78-85

30 Marcheggiani Muccioli GM, Grassi A, Setti S, et al. Conservative treatment of spontaneous osteonecrosis of the knee in the early stage: pulsed electromagnetic fields therapy. Eur J Radiol 2013;82 (03):530-537

31 Vitali M, Naim Rodriguez N, Pedretti A, et al. Bone marrow edema syndrome of the medial femoral condyle treated with extracorporeal shock wave therapy: a clinical and MRI retrospective comparative study. Arch Phys Med Rehabil 2018;99(05):873-879

32 Sansone V, Romeo P, Lavanga V. Extracorporeal shock wave therapy is effective in the treatment of bone marrow edema of the medial compartment of the knee: a comparative study. Med Princ Pract 2017;26(01):23-29

33 Gao F, Sun W, Li Z, et al. Extracorporeal shock wave therapy in the treatment of primary bone marrow edema syndrome of the knee: a prospective randomised controlled study. BMC Musculoskelet Disord 2015;16:379

34 Varenna M, Zucchi F, Failoni S, Becciolini A, Berruto M. Intravenous neridronate in the treatment of acute painful knee osteoarthritis: a randomized controlled study. Rheumatology (Oxford) 2015;54(10):1826-1832

35 Laslett LL, Doré DA, Quinn SJ, et al. Zoledronic acid reduces knee pain and bone marrow lesions over 1 year: a randomised controlled trial. Ann Rheum Dis 2012;71(08):1322-1328

36 Bartl C, Imhoff A, Bartl R. Treatment of bone marrow edema syndrome with intravenous ibandronate. Arch Orthop Trauma Surg 2012;132(12):1781-1788

37 Claßen T, Becker A, Landgraeber S, et al. Long-term clinical results after iloprost treatment for bone marrow edema and avascular necrosis. Orthop Rev (Pavia) 2016;8(01):6150

38 Zippelius T, Matziolis G, Perka C, Putzier M, Strube P, Röhner E. Long-term effects of intravenous iloprost therapy in patients with bone marrow oedema of the knee joint. Acta Chir Orthop Traumatol Cech 2018;85(01):17-21

39 Pountos I, Giannoudis PV. The role of iloprost on bone edema and osteonecrosis: safety and clinical results. Expert Opin Drug Saf 2018;17(03):225-233

40 Cohen SB, Sharkey PF. Surgical treatment of osteoarthritis pain related to subchondral bone defects or bone marrow lesions: subchondroplasty. Tech Knee Surg 2012;11:170-175

41 Farr J, Cohen SB. Expanding applications of the subchondroplasty procedure for the treatment of bone marrow lesions observed on magnetic resonance imaging. Oper Tech Sports Med 2013;21:138

42 Chatterjee D, McGee A, Strauss E, Youm T, Jazrawi L. Subchondral calcium phosphate is ineffective for bone marrow edema lesions in adults with advanced osteoarthritis. Clin Orthop Relat Res 2015;473(07):2334-2342

43 Collins NJ, Misra D, Felson DT, Crossley KM, Roos EM. Measures of knee function: International Knee Documentation Committee (IKDC) Subjective Knee Evaluation Form, Knee Injury and Osteoarthritis Outcome Score (KOOS), Knee Injury and Osteoarthritis Outcome Score Physical Function Short Form (KOOS-PS), Knee Outcome Survey Activities of Daily Living Scale (KOS-ADL), Lysholm Knee Scoring Scale, Oxford Knee Score (OKS), Western Ontario and McMaster Universities Osteoarthritis Index 
(WOMAC), Activity Rating Scale (ARS), and Tegner Activity Score (TAS). Arthritis Care Res (Hoboken) 2011;63(11, Suppl 11): S208-S228

44 Bonadio MB, Giglio PN, Helito CP, Pécora JR, Camanho GL, Demange MK. Subchondroplasty for treating bone marrow lesions in the knee - initial experience. Rev Bras Ortop 2017;52 (03):325-330

45 Chua K, Kang JYB, Ng FDJ, et al. Subchondroplasty for bone marrow lesions in the arthritic knee results in pain relief and improvement in function. J Knee Surg 2021;34(06):665-671
46 Laupattarakasem W, Laopaiboon M, Laupattarakasem P, Sumananont C. Arthroscopic debridement for knee osteoarthritis. Cochrane Database Syst Rev 2008;(01):CD005118

47 Thorlund JB, Juhl CB, Roos EM, Lohmander LS. Arthroscopic surgery for degenerative knee: systematic review and metaanalysis of benefits and harms. BMJ 2015;350:h2747

48 Brignardello-Petersen R, Guyatt GH, Buchbinder R, et al. Knee arthroscopy versus conservative management in patients with degenerative knee disease: a systematic review. BMJ Open 2017;7 (05):e016114 